\title{
Early Innervation of Skeletal Muscle During Tail Regeneration in Urodele Amphibians
}

\author{
YVES R. THOUVENY, THOMAS E. KOMOROWSKI, JEAN-PIERRE \\ ARSANTO, AND BRUCE M. CARLSON \\ Department of Anatomy and Cell Biology, Medical School, University of \\ Michigan, Ann Arbor, Michigan 48109 (T.E.K., B.M.C.) and Laboratoire \\ de Biologie de la Différenciation Cellulaire, UA 179 CNRS, Faculté des \\ Sciences de Luminy 13288, Marseille Cédex, France (Y.R.T., J.-P.A.)
}

\begin{abstract}
The innervation pattern of skeletal muscles was studied in the normal and regenerating tail of Notophthalmus viridescens. Silver staining for nerve endings and histochemical localization of acetylcholinesterase (AChE) were used for light microscopy. In normal musculature, AChE positive reactions were localized at the ends of the muscle fibers where they are anchored on connective tissue septa by myotendinous junctions. At this level, silver staining shows nerve terminals forming endplates. During regeneration, positive reactions for $\mathrm{AChE}$ appear de novo as dense plates localized at the ends of the newly formed myotubes. The mechanisms involved in the localization of $\mathrm{AChE}$ on this surface seem to operate before previous local contacts by nerve terminals. From the ultrastructural data and immunohistochemical results with anti-laminin antibody, these observations suggest that regenerating muscle fibers determine a region of post-synaptic specialization in close relation with the organization of myotendinous regions and basement membrane formation. Nerve-muscle contacts appear at these levels at stage IV (15-20 days after amputation) in the stump and in the rostral part of the regenerate (transition zone). These nerve terminals are provided by the disorganized peripheral nervous system of the injured segment. In the regenerate a similar pattern of $\mathrm{AChE}$ reaction can be seen in every myotube, differentiating according to a rostro-caudal gradient. Innervation at the ends of the muscle fibers is in spatiotemporal relation with the exits of the ventral roots from the regenerating nerve cord as the regenerate continues to grow in length.
\end{abstract}

An adult urodele amphibian can regenerate its tail after amputation by forming a blastema at the tip of the stump. This blastema gives rise to a regenerate in which cell differentiation occurs. Epidermal structures, skeleton, central (CNS) and peripheral (PNS) nervous systems, and skeletal muscles are regenerated in a normal structural pattern through five recognizable stages of which the main histological characteristics are summarized in Table 1, from the data of Iten and Bryant ('76a) and from our observations in respect to the shape and the days post-amputation of the regenerate. The number of segmented structures (myomeres and vertebrae) that form in the regenerate is directly proportional to the number removed by amputation (Iten and Bryant, '76a,b).

The evidence that two distinct modes of skeletal muscle regeneration exist was proposed by Cox ('69) studying muscle regeneration in the lizard tail and generalized by Carlson ('70) to the amphibian regeneration: 1) a tissue mode that re- stores the damaged muscle fibers in the injured myomeres at the level of amputation; and 2) an epimorphic mode that occurs within the regenerate itself. Mufti ('73), who first studied muscle regeneration in newt tail system, showed that the two processes of muscle regeneration are temporally and spatially distinct. Iten and Bryant ('76b) showed that a gradient in muscle differentiation exists from the stump to the regenerate. Only later do muscle masses segregate and display a longitudinal segmented pattern, forming new myomeres. At a given stage of regeneration, the most proximal muscle fibers are numerous and histologically mature, whereas myoblasts and myotubes are present in the distal growing part of the regenerate.

Regeneration of newt spinal cord occurs in a proximal-distal direction, with neurons of the stump sending their axons along the regenerating ependymal tube (Egar and Singer, '72). Some of

Received October 1, 1990; revision accepted May 14, 1991. 
TABLE 1. Main histological characteristics of tail regeneration ${ }^{1}$

Stage I 1 -6 days after amputation): wound healing
Migration of epidermis over the amputation surface
Breakdown of the distal muscle in the stump
Ependymal epithelium is closed off and ependymal vesicle is formed
Nerves from the spinal ganglia appear disorganized, ending at the amputation
level
Stage II 5 -11 days after amputation): blastema formation
In the stump, muscle dedifferentiates
Ependymal tube with terminal vesicle grows into the blastema
Peripheral nerve fibers grow in the regenerate and innervate the epidermis
Mesenchymal blastema cells begin to accumulate beneath the epidermis
Aggregates of myoblasts and myotube formation are seen in the stump and in the
lateral part of the rostral part of the regenerate
Cartilage rod differentiates under the spinal cord
AChE reaction appears at the ends of the myotubes and new basement membrane
is formed around them
Stage III $10-15$ days after amputation): cone-shaped blastema
Early stage IV:
Muscle fibers with cross striations and nerve-muscle contacts are visible in the
stump
Aggregates of myoblasts and bundles of myotubes with AChE reaction at their
ends are seen in the rostral part of the regenerate
Scattered aggregates of myoblasts are seen in the caudal part
Late stage VI:
Myogenesis continues in the distal part of the regenerate
Striated muscle fibers organize into myomeres isolated by myosepta
Spinal ganglia are formed and innervation of skeletal muscle fibers shows a
rostro-caudal gradient
Segmentation of the cartilage rod and formation of the neural and hemal arches
(30-60 days after amputation): growth
Myomeres with lateral and medial muscle fibers form along the length of the re-
generate
Stage
(13-22 days after amputation): elongation of the regenerate

${ }^{1}$ From Iten and Bryant (76a) and our results.

the growing axons exit from the regenerating spinal cord, forming ventral roots going to specific targets in the regenerate (i.e., regenerating muscle fibers). Nerves coming from the PNS of the injured body segment grow into the blastema, where they are suspected to exert a neurotrophic effect (Singer, '65; Brockes and Kintner, '86). Consequently, the blastema and early regenerate are very richly provided by nerve fibers. However, it is not known when and where nerve terminals make specific contacts (endplates) with the regenerating skeletal muscles.

During vertebrate development, contacts of nerve terminals are observed throughout the length of newly formed myotubes, corresponding to the deposition of $\mathrm{AChE}$, a marker of synapse formation. This scheme suggests that the initial sites of nerve-muscle contacts occur at random along the length of each myotube and that mature muscle fibers can subsequently eliminate incorrect synapses in favor of correct ones. But, it was also demonstrated, in vitro, that in the absence of axons, muscle cells may determine the formation of a post-synaptic organization (Moody-Corbett and Cohen, '82) and that, during regeneration, information about the synaptic site remaining in the muscle basement membrane controls the pattern of reinnervation (Sanes, '89, for review).

The present study was designed to determine the spatio-temporal sequence of muscle reinnervation during tail regeneration of Notophthalmus viridescens at the level of the stump (tissue mode of regeneration) and in the regenerate (epimorphic regeneration) in relation to the persistence and/or the formation of the basement membrane.

\section{MATERIALS AND METHODS \\ Animals}

Adult newts, Notophthalmus viridescens, were obtained from Lee's Newt Farm, Oak Ridge, Tennesee, and were maintained at $25^{\circ} \mathrm{C}$ on a diet of Tubifex. After anesthetization with 1:1,000 MS 222 (Sigma), amputations were performed 
through the proximal third of the tail at the level of the intervertebral junction. Operated animals were observed daily.

\section{Histology}

For histological study, blastemata of regenerates along with a few millimeters of the stump were removed, fixed in Bouin's fluid, decalcified in $0.5 \mathrm{M}$ EDTA (ethylenediamine tetraacetate), dehydrated in graded alcohols, and embedded in Paraplast. Serial sections were cut at $6 \mu \mathrm{m}$ and stained with hematoxylin and eosin or with Heidenhain's aniline blue technique for connective tissue. Serial sections cut at $15 \mu \mathrm{m}$ were stained with the reducing silver method of Samuel ('53) for the detection of nerve fibers. A total of 28 samples covering the five stages of tail regeneration were examined.

\section{Histochemistry}

For the detection of acetylcholinesterase (AChE) sites, samples were directly embedded unfixed in OCT (Miles Scientific, Naperville, IL) and frozen in 2-Methyl-Butane cooled with dry ice. Serial cryostat sections cut at $15 \mu \mathrm{m}$ were fixed and stained with the Goshgarian ('77) silver-AChE method for nerve fiber and endplate detection and counterstained with eosin. Twenty-eight regenerates corresponding respectively to stage II ( 7 samples), stage III (7 samples), early stage IV (7 samples), and late stage IV and stage V ( 7 samples) were used for this study.

\section{Electron microscopy}

According to the rostro-caudal gradient in the procress of differentiation, tissue samples of stage III and early stage IV were taken along the proximodistal axis of the regenerates and fixed for $2.5 \mathrm{hr}$ in $2.5 \%$ glutaraldehyde, $4 \%$ formaldehyde in $0.1 \mathrm{M}$ cacodylate buffer ( $\mathrm{pH} 7.4$ ), postfixed for $1 \mathrm{hr}$ in $1 \%$ osmium tetroxide in the same buffer, dehydrated in acetone, and embedded in epon. Alternate semi-thin $(1 \mu \mathrm{m})$ and ultrathin sections were collected. Semi-thin sections were stained with toluidine blue. Ultrathin sections were stained with uranyl acetate and lead citrate and examined with a Philips 400 or Hitachi $\mathrm{H} 600$ electron microscope.

\section{Immunohistochemistry}

For preparation of cryostat sections, samples were embedded in OCT compound. They were frozen rapidly in liquid nitrogen and stored at $-20^{\circ} \mathrm{C}$. Serial sections of $15 \mu \mathrm{m}$ were cut in a cryostat at $-22^{\circ} \mathrm{C}$, collected on polylysine-coated glass slides, and stored at $-20^{\circ} \mathrm{C}$ until immunofluorescent staining was performed. Anti-mouselaminin polyclonal antibody kindly provided by Dr. J.F. Riou and monoclonal antibody antineurofilaments (Immunotech, France) were used in double-labeling experiments. These antibodies were previously described respectively by Riou et al. ('87) and Alfonsi et al. ('83).

\section{RESULTS}

\section{Normal muscle organization and innervation in the tail}

The caudal musculature is formed by two types of axial muscles or myomeres, the medial musculature attached to the hemal or to the neural spines of adjacent vertebra and the lateral musculature attached to myosepta and to the transverse processes. The motor axons of the ventral roots pass through the spinal ganglia. Spinal nerves diverge backward to give rise to dorsal and ventral branches running into the connective tissue located between the medial and the lateral musculature. These branches divide and go through the myosepta until they reach the myofibers. Our major histological features confirm previous observations of Francis ('34) and Iten and Bryant ('76a) for musculature and those of Anton and Doring ('88) for the PNS.

With the Goshgarian technique we co-localized nerve fibers and AChE + sites (Figs. 1, 2). These results show that the motor endplates are located at the ends of the muscle fibers, where they are anchored to the connective tissue. The histochemical reaction is strongly positive at the apex and appears as a dense plate associated frequently

Fig. 1. Normal muscle. Longitudinal section of lateral musculature showing the sites of $\mathrm{AChE}$ reaction at the ends of muscle fibers (arrows), where they are anchored to the connective tissue. Note the myomeric organization of the muscle fibers (M). Silver-stained nerve fibers can be detected in the framed area (for enlargement see Fig. 2). Goshgarian method. Ms, myosepta. $\times 140$.

Fig. 2, Normal muscle. Framed area from Fig. 1. Nerve fibers (N) contacting sites of AChE reaction (arrowhead) and positive synaptic dots on the lateral surface (arrows) of muscle fibers can be observed. Goshgarian method. $\times 750$.

Fig. 3. Normal muscle. High magnification of end of muscle fiber (M) showing the anchoring AChE-positive zone (arrowheads), lateral AChE-positive dots (arrows), and nerve fibers (N). Goshgarian method. C, connective tissue matrix $\times 1,000$ 


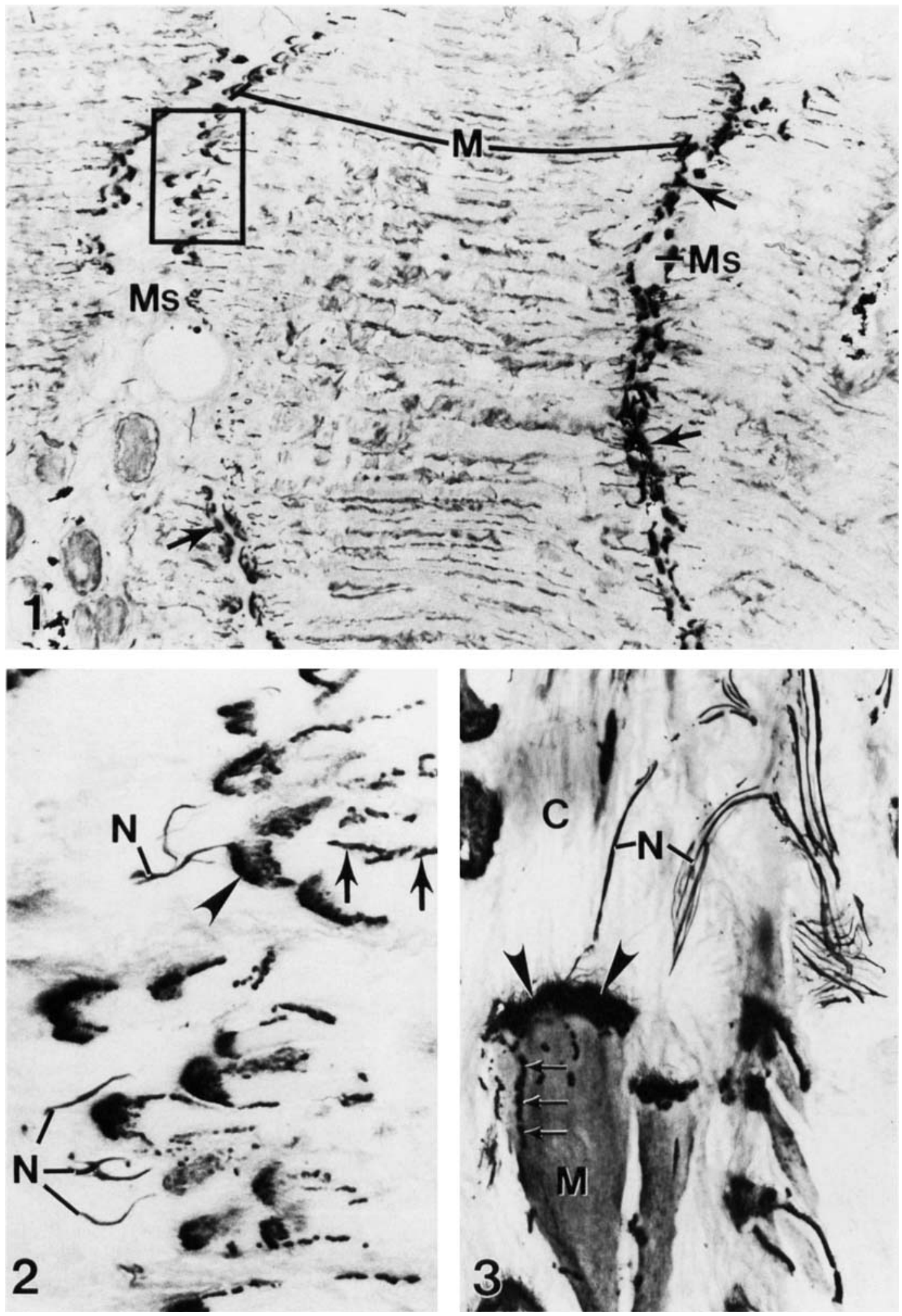

Figures 1-3. 

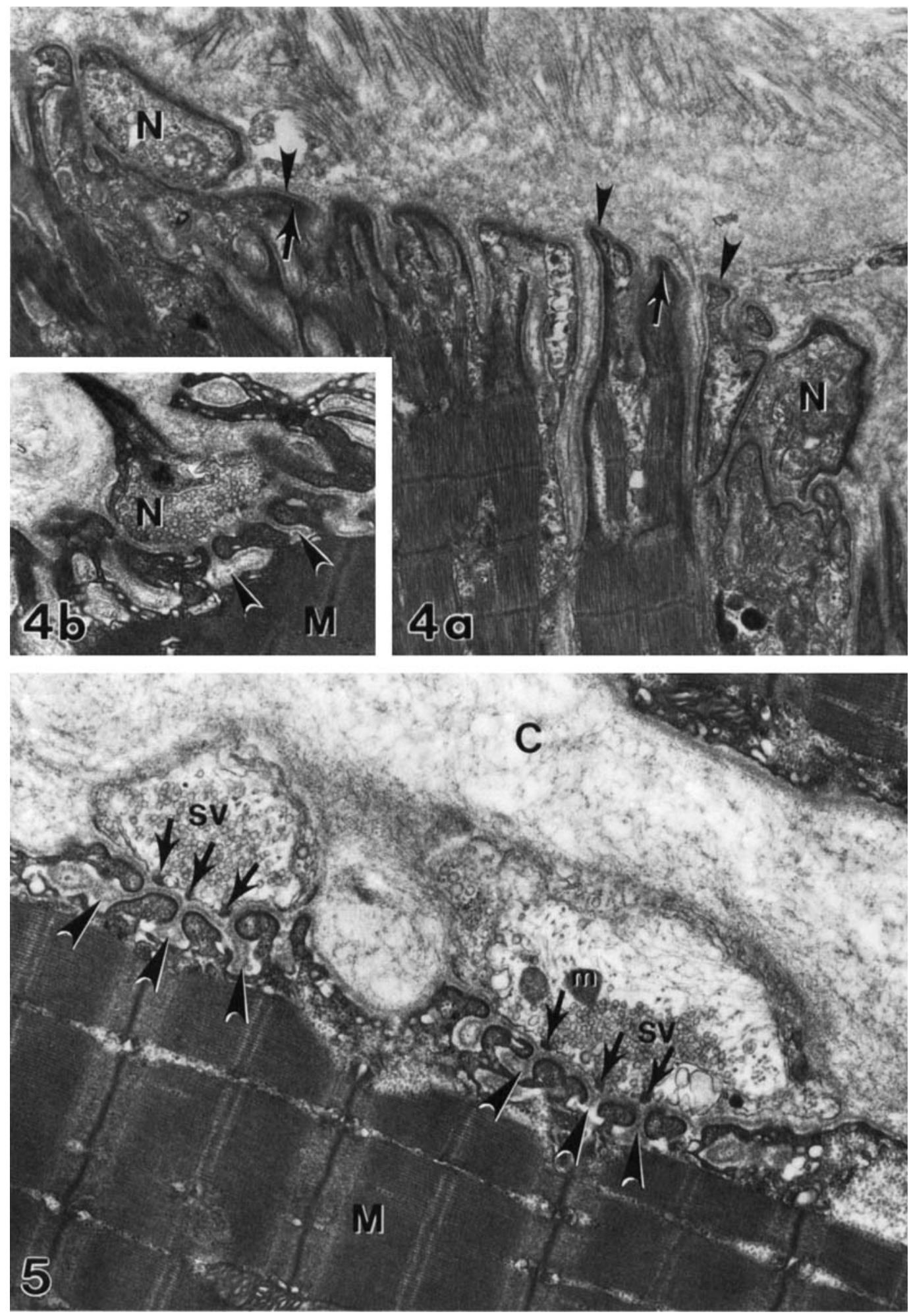

Figures 4 and 5. 
with tufts of extracellular material. Dots of reaction product are also visible along the lateral surface (Fig. 3). No reaction can be observed along the medial part of the fiber.

Electron micrographs (Fig. 4a,b) of the ends of the muscle fibers show that the plasmalemmal membrane forms numerous projections penetrating deeply into the extracellular matrix. Among these projections, axons can frequently be observed. They establish neuromuscular contacts identifiable by the characteristic accumulation of vesicles in the presynaptic bud and by the presence of subneural clefts on the muscle fiber. Endplates can also be observed on the lateral surface near the ends of the muscle fiber (Fig. 5). They appear as a series of nerve-muscle contacts formed by the same nerve fiber creeping along the sarcolemma. In this figure a tangential section of the plasmalemma and surrounding dense material located between two consecutive pre-synaptic buds is shown.

\section{Nerve muscle relationships during early stages of regeneration at the level of the stump}

A few days after amputation, a disorganization of severed muscles can be seen in the stump. The endomysium and the perimysium are altered in their morphology and muscle masses are replaced by a loose connective tissue in which numerous fragments of degenerating muscle fibers, fibroblast-like cells, macrophages, and blood cells can be observed. These observations are consistent with those of Mufti ('73) and Iten and Bryant ('76). We will only report observations concerning most interesting stages (stages III and IV) in muscle regeneration and innervation.

\footnotetext{
Fig. 4. a: Normal muscle. Electron micrograph showing the anchoring end of the muscle fiber and the presence of nerve fibers $(\mathrm{N})$ containing synaptic vesicles. Note the presence of dense material (arrows) accumulated on the inner part of the sarcolemma and of the basement membrane (arrowheads). $\times 14,600$. b: Normal muscle. Typical motor endplate seen at the end of a muscle fiber $(\mathrm{M})$. N, nerve; arrowheads, subneural clefts. $\times 19,000$.
}

Fig. 5. Normal muscle. Lateral endplates near the end of a muscle fiber (M). Tangential section of the plasmalemma and surrounding dense material located between two consecutive nerve terminals suggest the continuity of the nerve fiber. Note the presence of active sites (arrows) above the subneural clefts (arrowheads). C, connective tissue matrix; $\mathrm{m}$, mitochondrion; $\mathrm{SV}$, synaptic vesicles. $\times 19,000$.

\section{Stage III}

By stage III (10-15 days after amputation) a transition zone is located between the stump and the blastema. The mesenchyme of this zone is formed by the accumulation of undifferentiated cells. In front of this zone prefusion myoblasts showing the characteristic alignment and some sparse myotubes, oriented parallel to the surface of the epidermis, appear in the loose connective tissue of the stump (Fig. 6). They are involved in the regeneration of the musculature of the injured segment. The newly formed muscle fibers can be distinguished from the old ones by a reduced diameter and the central position of their nuclei.

Silver staining shows that the stump is supplied by numerous nerve fibers coming from the peripheral nervous system of the injured segment (Fig. 7). Bundles of nerve fibers run towards the amputation level and penetrate the regenerate. Other fibers radiate inside the loose connective tissue where they seem to proceed freely among regenerating myotubes without precise contacts with the new muscle fibers (Fig. 8). Co-localization of nerve endings and $\mathrm{AChE}$ sites show that, by stage III (10-15 days after amputation), a positive reaction for $\mathrm{AChE}$ appears as a dense plate covering the ends of the differentiating myotubes and myofibers. At this stage no patches of reaction are seen on the lateral surface and no nerve endings are revealed at this level.

Electron microscopy shows inside the loose connective tissue a ruffled granular layer corresponding to remnants of the old muscle basement membrane (Figs. 9, 10). Numerous threads of this dense material and winding profiles are frequently observed not directly in contact with the differentiating myotubes but, in Figure 9, one of them is completely included in a such winding process.

In differentiating myofibers a thin extracellular material is frequently seen in close apposition to the sarcolemma. This new basement membrane first appears at the ends of the muscle fibers where it is denser and thicker than on the lateral surface (Figs. 17, 18).

On immunohistochemically stained sections by anti-laminin antibody, the degenerating zone of musculature shows a ruffled reaction with respect to the regular profile of reaction in normal musculature (Figs. 12, 13a,b). The newly differentiating myofibers are surrounded by a slight reaction sometimes associated with discontinuous anti-laminin positive patches or threads (Fig. 14). 

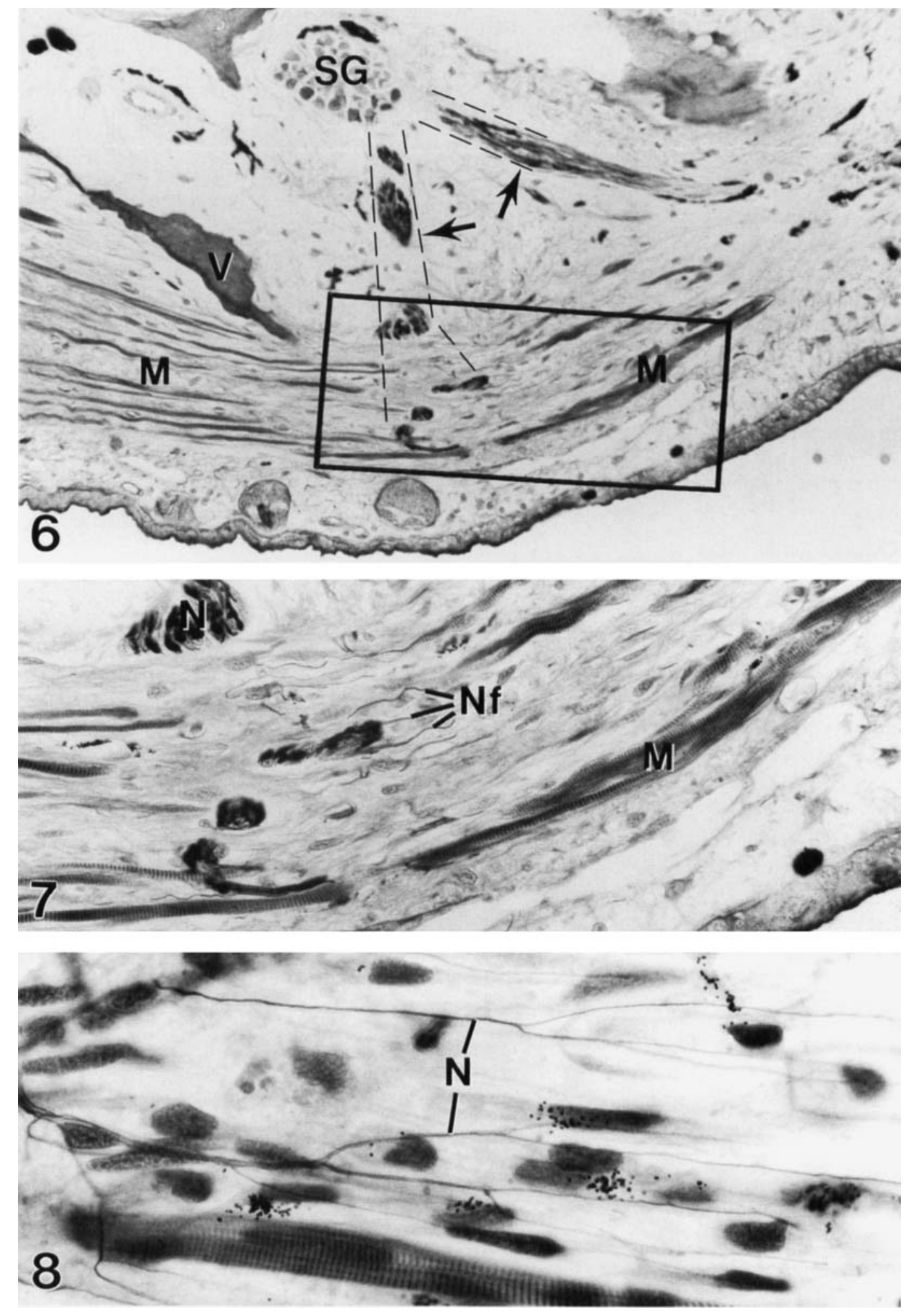

Figures 6-8. 
These observations suggest that the old basement membrane of the degenerating myofibers could guide the alignment of the prefusing myoblasts but is removed afterwards and replaced by a basement membrane formed de novo.

\section{Stage IV}

By stage IV (20 days after amputation), the $\mathrm{AChE}+$ reaction is identical to that of normal muscle in well-differentiated muscle fibers, and nerve endings are seen in close contact with the ends of the muscle fibers (Fig. 20). Patches of reaction are seen only at these ends. A good correlation exists between the appearance of the new basement membrane at these sites and the localization of the newly formed endplates (Fig. 19). A closely associated nerve fiber with numerous synaptic vesicles and mitochondria is seen in the connective tissue near the surface of the muscle fiber and on the neighboring lateral surface of the muscle fiber, and on the neighboring lateral surface, differentiating endplates can be seen.

\section{Nerve-muscle relationships within the regenerate during early stages of regeneration}

\section{Stage III}

By stage III (10-15 days after amputation), clusters of pre-muscle aggregates and bundles of myotubes are formed in the lateral region within the rostral part of the regenerate. They are frequently located at some distance from the end of the altered muscle masses of the stump. Electron microscopy shows that myotubes and/or myofi-

Fig. 6. Regenerating muscle. Parasagittal section through the regenerating zone of the stump at stage III, 15 days after amputation. Regenerating muscle fibers (M) are seen in the loose connective tissue. Two branches of the spinal nerve are visible (arrows), one going to regenerating muscle of the stump, the other going to the regenerate at the right of the figure. The continuity of the spinal nerve branches, visible on serial sections, is indicated by the dashed lines. Note the presence of spinal ganglia (SG) and section of a vertebra $(\mathrm{V})$ of the injured segment. Silver-stained sections counterstained with eosin. $\times 250$.

Fig. 7. Enlargement of the framed part of Fig. 6, showing nerve fibers (Nf) ending in the connective tissue. $M$, regenerating muscle fibers; N, nerve fibers. Silver-stained section, counterstained by eosin. $\times 500$.

Fig. 8. Another view of the richly innervated regenerating muscle of the stump. Numerous nerve fibers $(\mathrm{N})$ are observed among regenerating myoblasts and myotubes. Silverstained section counterstained by eosin. $\times 1,500$. bers are provided with a layer of extracellular material directly in contact with the sarcolemmal membrane. This newly formed basement membrane is more dense at the ends of the fibers than on the lateral surface. Immunoreactivity with anti-laminin antibody confirms this observation. In Figure 15, the ends of muscle fibers appear more densely labeled by the antibody than the lateral surface (double arrows). AChE sites are located at the level of the ends of the differentiating muscle fibers without apparent nerve-muscle contacts (Fig. 21). Indeed, the regenerate is supplied by numerous nerve fibers coming from the PNS of the stump. Nerve bundles are frequently seen running parallel to the myogenic zone and are visible up to the epidermis. But no focal contacts were distinctly observed on serial sections, at the light or at the EM level.

Immunohistochemical staining with neurofilament monoclonal antibody used in double-labeling experiments with anti-laminin antibody (Fig. 16) confirms our observations with the Goshgarian technique and with E.M. on the absence of nerve-muscle contacts.

\section{Stage IV}

As early as stage IV (15-20 days after amputation), myofibers are visible at this level and they are already distinctly arranged segmentally as myomeres. This disposition foreshadows the segmentation pattern of the regenerated tail. However, at this stage, boundaries between myomeres are not yet distinctly established as myoseptal connective tissue.

Silver-stained sections show that numerous nerve fibers are present in the ventro-lateral part of the ependymal tube. In sagittal sections, these fibers are seen in continuity with those of the spinal cord of the stump (not shown). In some places along the rostro-caudal axis, nerve fibers exit from the ependymal tube and form the ventral root anlagen running until the myogenic zone (Fig. 22). Taking advantage of the existence of a rostro-caudal gradient of regeneration of the spinal cord and of the exit of the ventral roots, it was possible to observe the spatio-temporal progress of the innervation with the regenerate.

Electron microscopy shows that, at the emergence of the ventral roots, exiting nerve fibers are wrapped by glio-ependymal cell processes (Fig. 23), as previously described by Nordlander and Singer ('78) and Géraudie et al. ('89). On serial ultrathin sections we showed nerve processes wrapped by Schwann cells running laterally into 

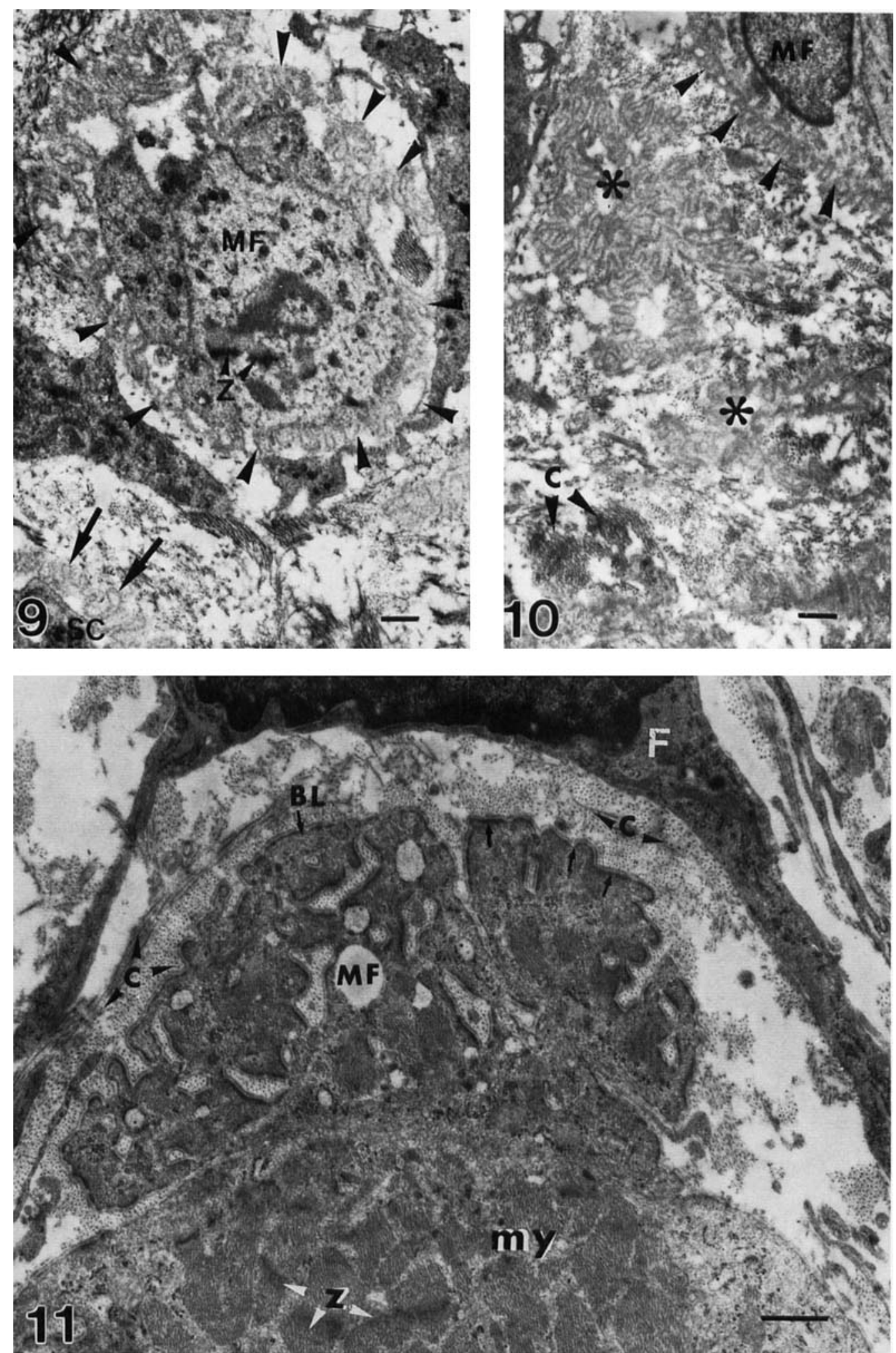

Figures 9-11. 
the connective tissue densely populated by collagen fibers. At the level of the regenerating myofibers, early developmental stages of motor endplates can be observed (Figs. 24, 25). The axonal terminus is dilated and contains small mitochondria and a large number of synaptic vesicles. Schwann cell cytoplasmic processes are absent on the side of the axon facing the differentiating muscle fibers, but they frequently cover the opposite surface.

On the muscle surface, invaginations of the plasmalemma containing a dense extracellular material mark the first appearance of the subneural clefts. The forming endplates can be detected at the time when the myofibers exhibit numerous striated myofibrils. The number of such structures characteristic of motor nerve-muscle contacts increases in the rostral direction.

\section{DISCUSSION}

In this paper, we describe the patterns of reinnervation of skeletal muscle during post-traumatic regeneration of the tail of the urodele amphibian Notophthalmus viridescens. Our results are as follows.

Reinnervation of the regenerating muscle first appears at an early stage, 15-20 days after amputation (early stage IV of Iten and Bryant, '76a). The innervation pattern is different depending on the level of the regenerating part. At the level of the stump and in the transitional zone located in the proximal part of the regenerate, nerve endings originate from the PNS of the injured body segment. Although it was not possible to distin-

Figs. 9, 10. Electron micrographs of parts of transversely sectioned damaged muscle fibers (MF) of the transition zone close to the amputation level, 15 days after amputation (stage III). Note the ruffled feature of the basal lamina sheath (arrowheads) of the dissociating myofibers, but also of that (arrows) of the Schwann cell only seen in part in the lower left (corner) in Fig. 9. Note also the aggregates of pleated basal lamina sheaths (asterisks) among extracellular matrix material rich in fibrillar collagen (c). SC, spinal cord; Z, Z band. In the two figures, bar equals $1 \mu \mathrm{m}$. Fig. 9, $\times 700$; Fig. $10, \times 7,000$.

Fig. 11. Electron micrographs of a cross-section through an area of regenerated muscle fibers (MF) in the rostral part of a 21 day old tail blastema. The plane of section nearly coincides with the endplate region of the myofibers, which is also their insertion zone where the basal lamina (BL) is particularly well visible. Note the highly stained sites attached to the myofibrils. Note also the presence of a fibroblast $(\mathrm{F})$ close to the muscle fibers. The cytoplasmic processes of this fibroblast are most likely involved in the synthesis of collagen fibrils (c) of the forming perimysial sheath. my, myofibrils; $Z, Z$ band. Bar equals $1 \mu \mathrm{m} . \times 1,000$. guish between sensory and motor fibers, it appears that by stage III, many fibers of spinal nerves remain located at this level and extend through the loose connective tissue in the region of the degenerating muscle masses. By stage IV, nerve fibers establish contacts with the ends of the differentiating muscle fibers. In the regenerate, the most characteristic feature of the innervation of the epimorphically regenerating muscle is the appearance of the ventral roots coming from the regenerated spinal cord, already observed by previous investigators (see Géraudie et al., '89, for review). The temporally correlated differentiation of endplates is very rapid and follows a rostro-caudal gradient. Another potential source of innervating axons comes from the spinal roots of segments rostral to the amputation plane. We observed such nerve fibers running in the myogenic zone but no focal contacts between them and regenerating muscle fibers were seen at these early stages of regeneration (cf. Fig. 16 and section 3, below).

The presence of the old basement membrane in the loose connective tissue of the stump confirms the existence of the tissue mode of muscle regeneration in the stump, described by Carlson ('70) and Mufti ('73). However, this structure is altered in its morphology, degenerated, and is replaced by a new one, directly in contact with the sarcolemmal membrane of the regenerating muscle fibers. The appearance of the new basement membrane progresses from the ends to the median part of the myotube. When the myofiber is mature, the new basement membrane is completely reformed and the remnants of the old one are not visible in their proximity. At the level of the epimorphically regenerating muscle fibers, the new basement membrane is formed de novo in close contact with the differentiating myotubes and as in the stump, a first layer of dense extracellular matrix appears at their ends and seems to progress in the direction of their median part.

These observations are in good agreement with those of Gulati et al. ('83a,b) who first studied changes in basement membrane zone components by immunohistochemistry, during skeletal muscle fiber regeneration in autotransplanted muscle in the rat and during epimorphic limb regeneration in the newt. In rat, during regeneration by the tissue mode, these investigators observed that the basement membrane is not a static structure as previously thought but rather that its components change rapidly during myofiber regeneration. During epimorphic regeneration in the 

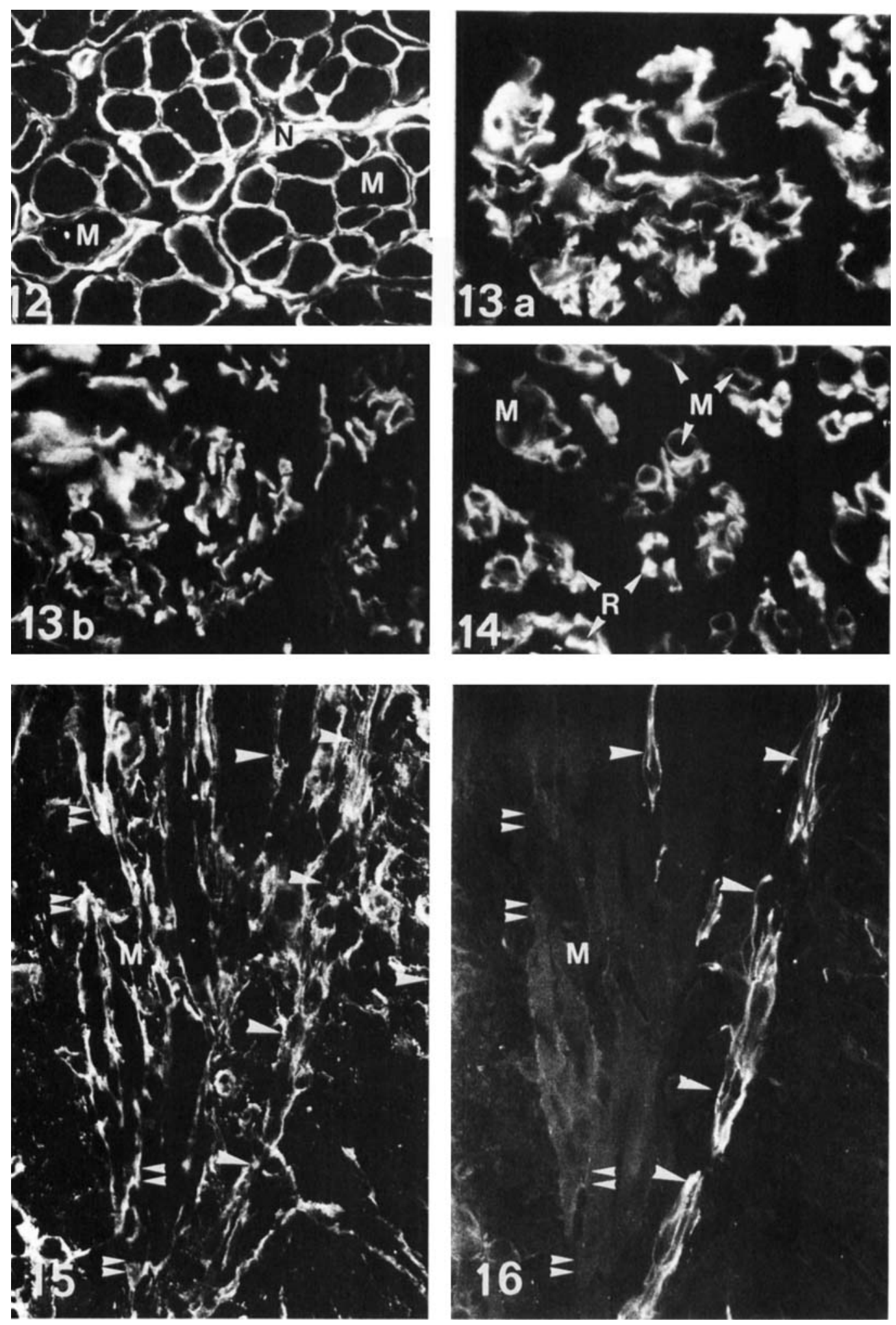

Figures 12-16. 
newt, they observed that laminin is seen for the first time around the newly regenerated myotubes in the region corresponding to their basement membrane. Our observations of tail regeneration suggest that the ruffled remnants of the old basement membrane, during the first stages of tissue mode regeneration in the stump, determine the alignment of prefusing myoblasts. However, as a new basement membrane is formed around the myotubes, the old one cannot be involved in the localization of the site of reinnervation of the myofibers.

$\mathrm{AChE}$ reactivity appears de novo at the ends of the muscle fibers. The reaction first appears as a dense plate with numerous fibrils radiating into the connective tissue of the myosepta. When nerve endings make contacts with the muscle fibers, they form small patches aligned on the lateral surface at the ends of the muscle fibers, near the terminal dense plates. Such dispositions are clearly seen in normal musculature and can be seen reappearing on regenerating muscle fibers. But the terminal dense plate is already observed before neuromuscular contacts are seen.

The nerve-muscle contacts are seen to be formed exclusively at the ends of the regenerating muscle fibers both in the stump and in the regenerate and there is a spatial correlation between the pattern of their formation, the pattern of $\mathrm{AChE}$ reaction, and the pattern of formation of the new basement membrane.

Fig. 12. Distribution of laminin in a cross-section of normal muscle. Laminin is seen in the endomysium of skeletal muscle $(\mathrm{M})$ and the perineurium of the nerve $(\mathrm{N}) . \times 120$.

Fig. 13. a,b: Distribution of laminin in a cross-section of the degenerating muscle in the stump, anterior to the level of the amputation, 10 days after amputation (stage II). Note the ruffled aspect of the reaction in respect to the normal musculature. $\times 120$.

Fig. 14. Distribution of laminin in the regenerating musculature, 15 days after amputation (stage III), in the stump. Note the presence of circular profiles (M) corresponding to the regenerated myofibers and threads $(\mathrm{R})$ corresponding to remnants of old basement membrane. $\times 120$.

Figs. 15, 16. Distribution of laminin and neurofilaments in the myogenic zone of the anterior part of the regenerate (longitudinal section), 15 days after amputation. Compared with Fig. 16 (double labeling with anti-laminin and antineurofilament antibodies). Note the presence of a dense reaction at the ends of the myotube (double arrowheads). The pathway of the nerves coming from the stump is labeled by the anti-laminin antibody (single arrowheads). In Fig. 16, these nerves do not contact the myofibers $(M) . \times 500$.
It has been demonstrated that adult salamander muscle has the ability to eliminate incorrect synapses in favor of correct ones (Dennis and Yip, '78). Such a selective process could also be involved in eliminating nerve fibers coming from the tail stump that do not correspond to the normal pattern of innervation. We have not observed the appearance of transitory positive reactivity for AChE. Furthermore, nerve endings contacting regenerating muscle fibers were seen only at the ends. Finally, we did not see the regression of foreign positive sites for $\mathrm{AChE}$ along the entire length of the muscle fiber as was observed by Bennett et al. ('79) in limb muscle of the axolotl.

During development and regeneration of the tail of the lizard Hemidactylus bowringi, Liu and Maneely ('68) showed that the AChE reaction appears on the muscle fibers close to the intersegmental zones before neural elements invade the developing muscle segment and long before they have differentiated endplates. The binding of $\mathrm{AChE}$ to the basement membrane is independent of nerves, as was demonstrated in vitro with amphibian myotubes by Moody-Corbett and Cohen ('82) and Lappin and Rubin ('85). In their studies, localization of $\mathrm{AChE}$ and also clusters of $\mathrm{AChR}$ are linked to an ultrastructural post-synaptic-like specialization, even in the absence of nerves. In vivo, myotendinous junctions of myotomal muscles of Xenopus laevis are sites of AChE activity (Cohen, '80). Our observations are in good agreement with the findings of these previous investigators. We first observed the appearance of dense positive plates at the ends of the muscle fibers during regeneration before nerve endings contact them.

The localization of AChE activity as a dense plate at the ends of the muscle fibers in normal and in regenerating muscle fibers raises the question of its specificity as an exclusive marker of the motor endplate (Massoulié and Bon, '82). Such localization might represent a phenomenon quite distinct from endplate localization concerning the molecular form of $\mathrm{AChE}$, as was reported in frog muscle lamina sheaths (Nicolet et al., '87) and in mammalian skeletal muscle (Garcia et al., ' 88 ). According to Wallace et al. ('85), the structural as well as the biochemical specialization of the basement membrane of the myotubes and/or extracellular matrix in this region could promote, in vivo, the formation of patches of $\mathrm{AChE}$ and the subsequent establishment of nerve contacts according to the metameric pattern of the caudal musculature and innervation. 

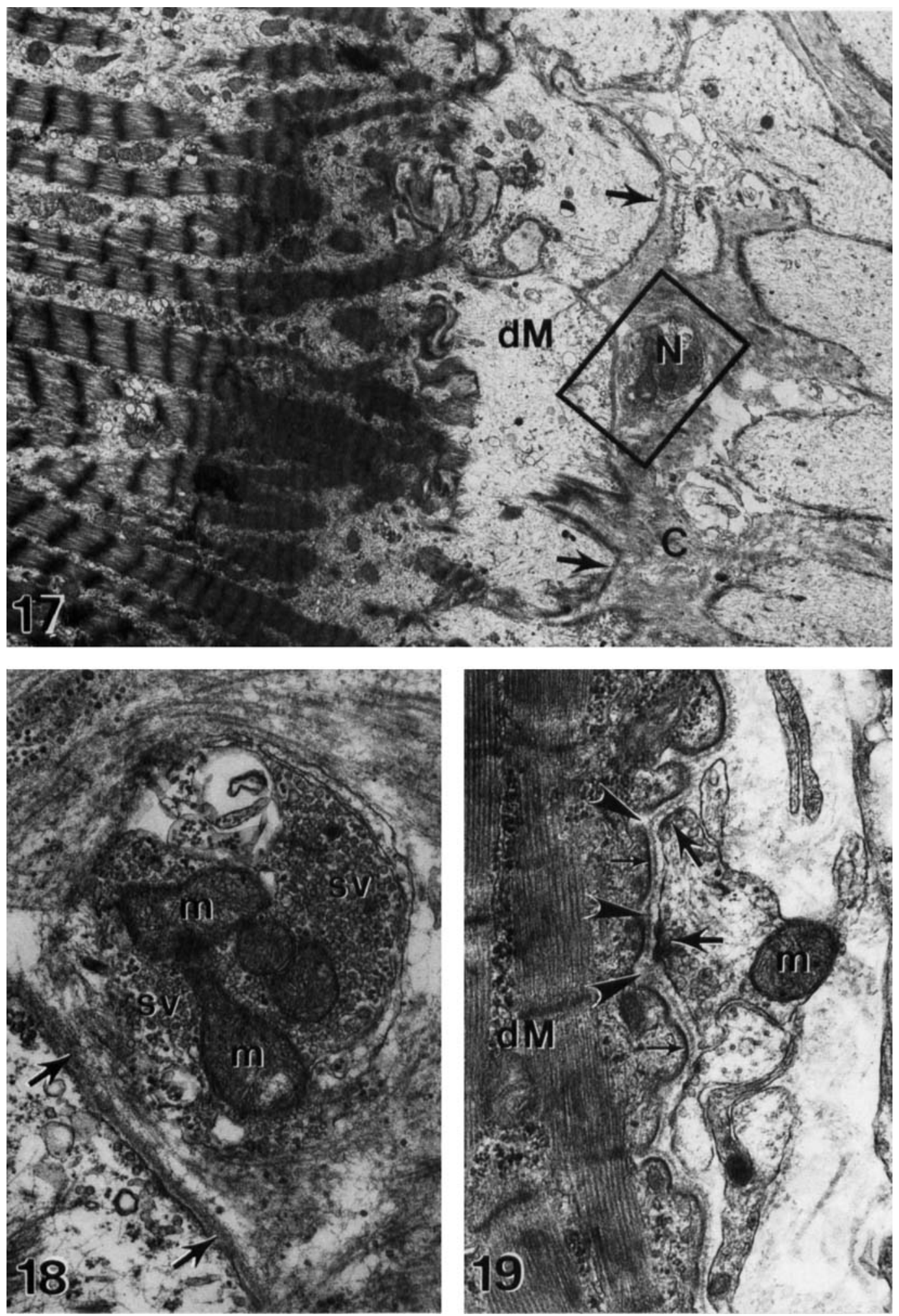

Figures 17-19. 


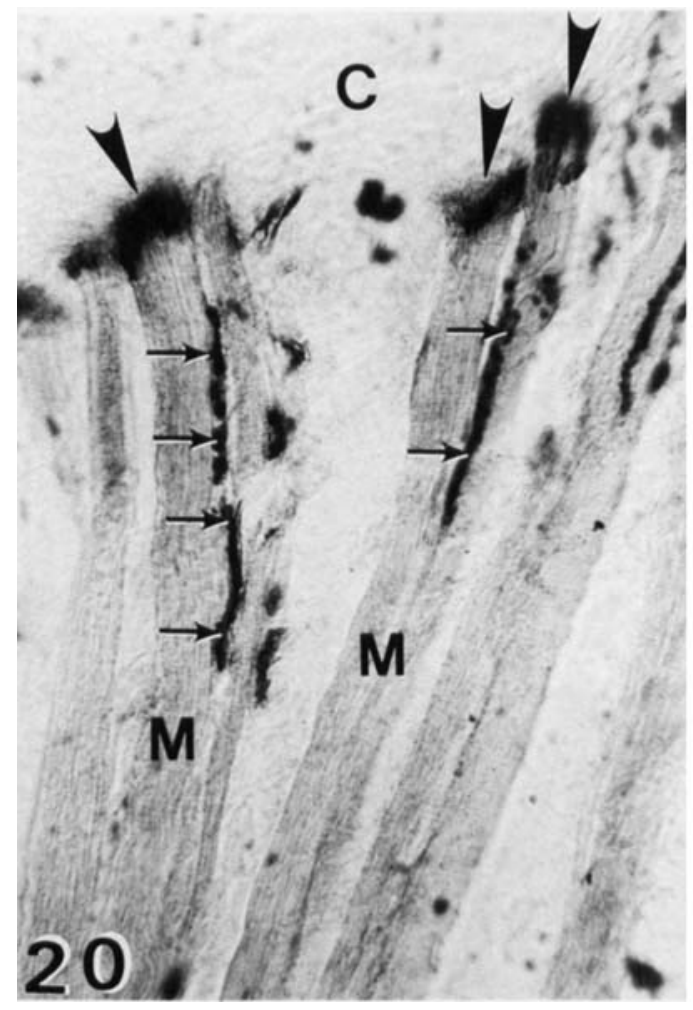

Fig. 20. AChE reaction in regenerating muscle fibers of the stump (stage IV), 20 days after amputation. Note the dense reaction (arrowheads) on the ends and lateral surface (arrows) of the muscle fibers (M) at this stage. These lateral dots correspond to the creeping of nerve fiber on muscle fiber. $\mathrm{C}$, connective tissue matrix. $\times 750$.

Fig. 17. Electron micrograph of the end of a differentiating muscle fiber (dM), 15 days after amputation (stage III), in the stump. Projections of the sarcolemma (arrows) begin to form in the extracellular matrix, and nerve fibers $(N)$ are in close contact with the muscle cell membrane. $\mathrm{C}$, connective tissue matrix. $\times 5,500$.

Fig. 18. Enlargement of the framed part of Fig. 17. Mitochondria $(\mathrm{m})$ and numerous synaptic vesicles (sv) are seen in the nerve ending. A basement membrane (arrows) is visible between the nerve ending and the differentiating muscle fiber. $\times 26,400$.

Fig. 19. Electron micrograph of a differentiating lateral motor endplate in the stump, 21 days after amputation (stage IV). Vesicles and active centers (arrows) are seen in the nerve terminal. Accumulations of dense material on the inner part of the sarcolemma (small arrows) are visible. Note the presence of forming subneural clefts (arrowheads) in front of the active centers. $\mathrm{dM}$, differentiating muscle fiber; $\mathrm{m}$, mitochondria. $\times 29,700$.

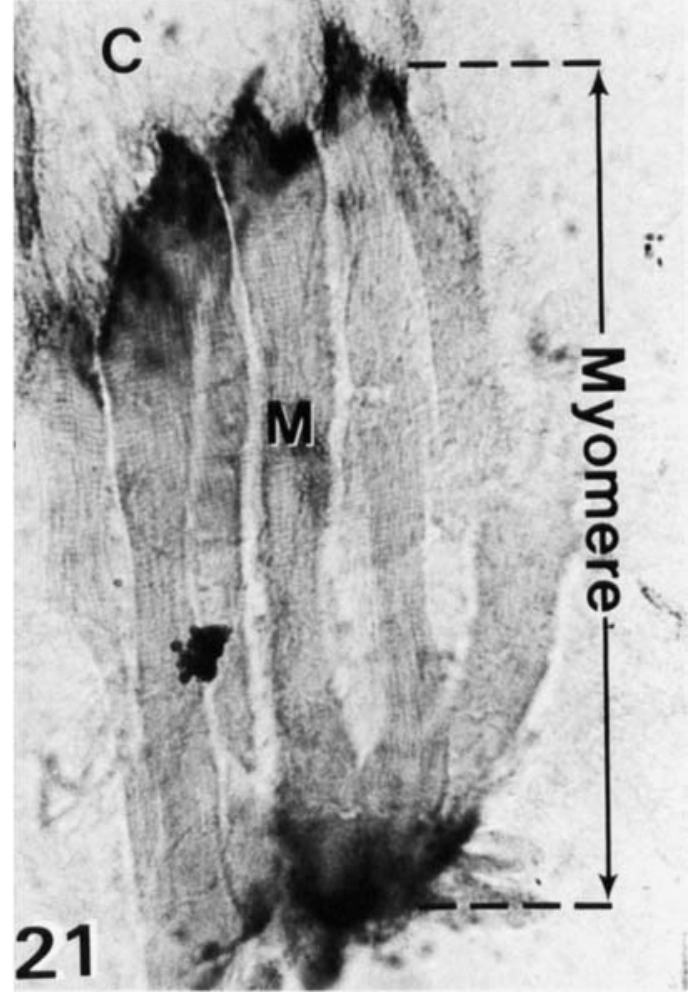

Fig. 21. Segment of regenerated musculature in a regenerate, 15 days after amputation (stage III), stained for AChE activity. The muscle fibers (M) are aligned, and the AChE reaction is concentrated at the ends of the muscle fibers, thus defining the organization of a new myomere between connective tissue septa (C). This muscle organization is seen in the regenerate. No nerve-muscle contacts are seen. No nerve contact can be detected in these fibers. $\times 750$.

Concerning the extracellular matrix, we previously reported the distribution of tenascin immunoreactivity in the myogenic zone during tail regeneration of the amphibian urodele Pleurodeles waltl (Arsanto et al., '90). We showed that the expression of this molecule is progressively restricted to the myoseptal connective tissue on which muscle fibers are anchored. Further studies on organizing processes of the extracellular matrix and basement membrane in this region during regeneration with antibody tools are in progress in the laboratory and will provide new information on the origin and the guidance of nerve endings and on the stabilization of nervemuscle contacts at this level.

\section{ACKNOWLEDGMENTS}

This investigation was supported by N.I.H. grant P01 DE 07687 and by the Association Française contre les Myopathies. 

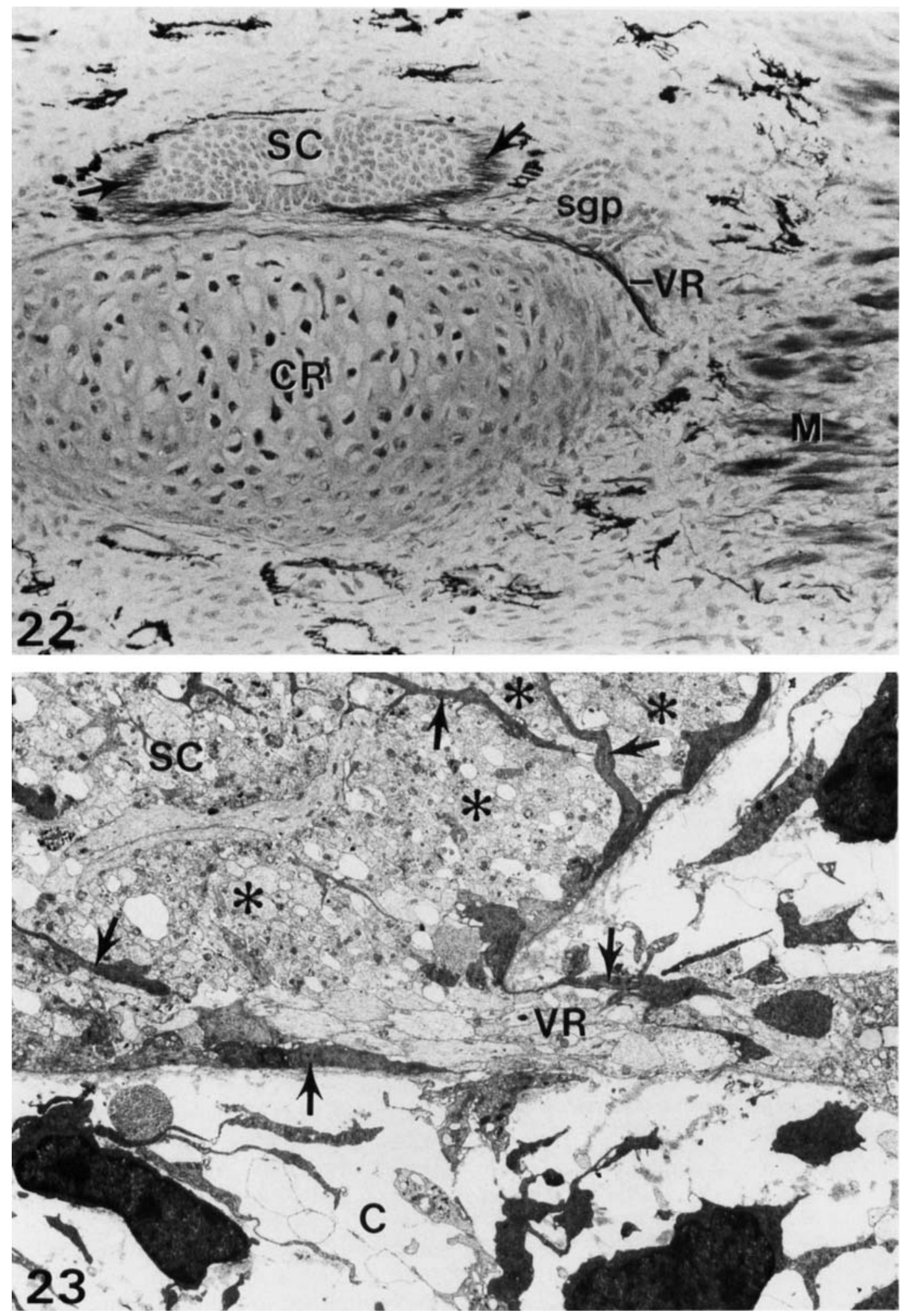

Fig. 22. Transverse section at the level of the regenerate (stage IV) 20 days after amputation. This section shows the exit of nerve fibers from the ventral part of the regenerating spinal cord (SC). The section is slightly oblique and shows the nerve tract (arrows) in the lateral wall of the ependymal tube. At this stage no neuronal cell bodies can be detected at this level. The ventral root (VR) can be seen in serial sections to enter the myogenic zone. Silver-stained section counterstained with eosin. CR, cartilage rod; M, muscle fibers; sgp, spinal ganglion primordium. $\times 500$.
Fig. 23. Electron micrograph at the level of the exit of the ventral root (stage IV). The corresponding semi-thin section (not shown) is similar to Fig. 9. Note the presence of numerous cross-sections of nerve fibers $\left({ }^{*}\right)$ wrapped by large expansions of ependymal cells (arrows) up to the periphery of the spinal cord (SC). C, connective tissue matrix; VR, ventral root. $\times 3,100$. 

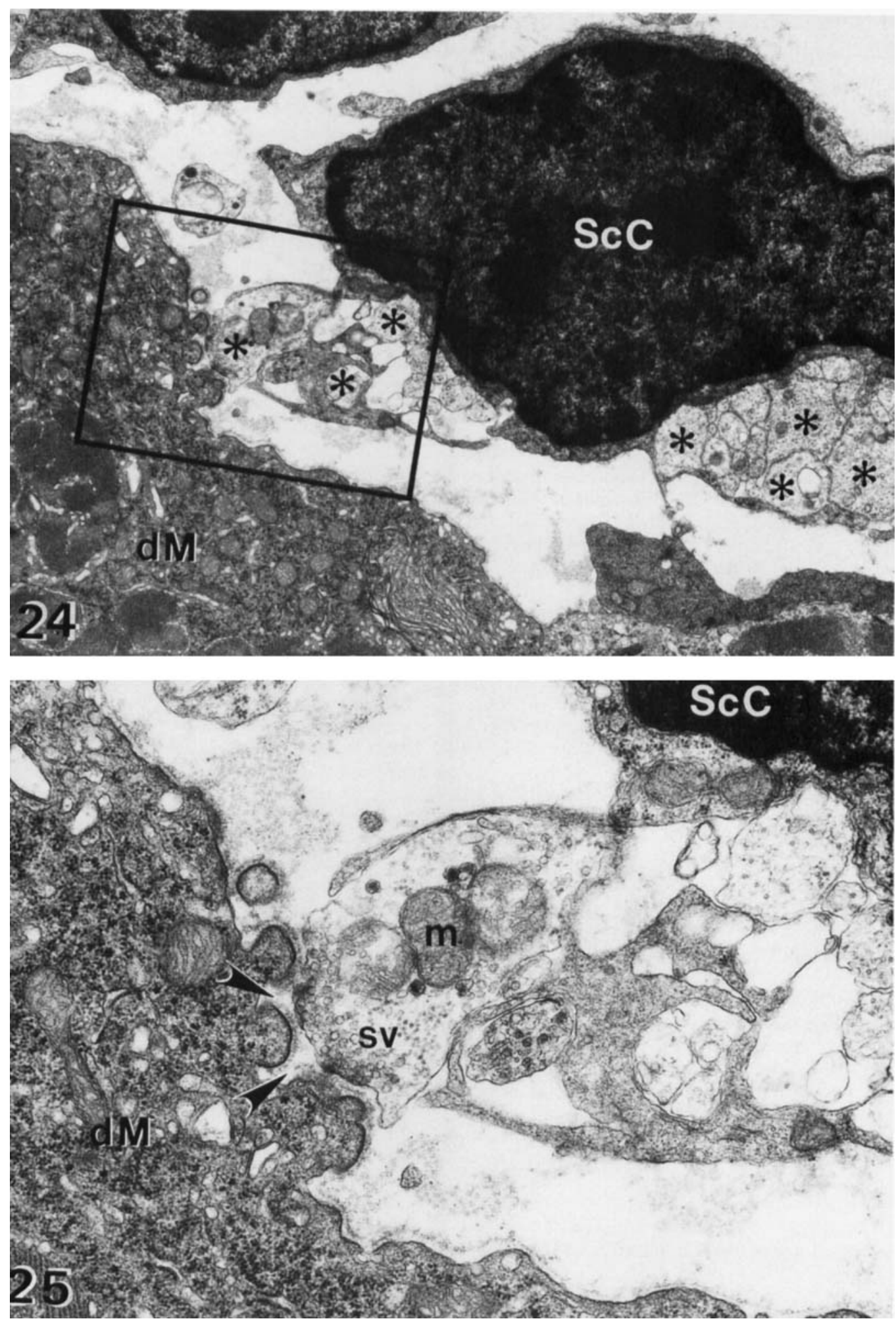

Fig. 24. Electron micrograph of a forming motor endplate in regenerate, 21 days after amputation (stage IV). Numerous nerve fibers $\left({ }^{*}\right)$ are visible in the connective tissue. Here they are wrapped by Schwann cell $(\mathrm{ScC})$ and make contact with a differentiating muscle fiber $(\mathrm{dM}) . \times 10,100$.
Fig. 25. Enlargement of area shown in Fig. 24. Note the formation of subneural clefts (arrowheads) on the differentiating muscle fiber (dM). ScC, Schwann cell; sv, synaptic vesicles; $\mathrm{m}$, mitochondrion. $\times 27,500$. 
During his sabbatical period Y.R. Thouveny found much hospitality and generosity in Dr. Bruce M. Carlson's laboratory. He wishes to thank Beverly Shepard and Jacqueline Rodgers for their expert assistance and the Fondation pour la Recherche Médicale Française for its help.

\section{LITERATURE CITED}

Alfonsi, F., M. Darmon, N. Forest, and D. Paulin (1983) Intermediate sized filaments as markers of neuronal differentiation. In: The Role of Cell Interactions in Early Neurogene. A.M. Duprat, A. Kato, and M. Weber, eds. Plenum Publ. Corp. N.Y., pp. 157-176.

Anton, H.J., and N. Doring (1988) Restitution of spinal ganglia during tail regeneration in Triturus. Monogr. Devel., 21:96-104.

Arsanto, J.P., M. Diano, Y. Thouveny, J.P. Thiery, and G. Levi (1990) Patterns of tenascin expression during tail regeneration of the amphibian urodele Pleurodeles Waltl. Development 109:177-188.

Bennett, M.R., P.A. Grath, and D.F. Davey (1979) The regression of synapses formed by foreign nerve in mature axolotl striated muscle. Brain Res., 173:451-469.

Brockes, J.P., and C.R. Kintner (1986) Glial growth factor and nerve dependent proliferation in the regeneration blastema of urodele amphibian. Cell, 45:301-306.

Carlson, B.M. (1970) Relationship between tissue and epimorphic regeneration of muscle. Am. Zool., 10:175-186.

Cohen, M.W. (1980) Development of an amphibian neuromuscular junction in vivo and in culture. J. Exp. Biol., 89: $43-56$.

Cox, P.G. (1969) Some aspects of tail regeneration in the lizard, Anolis carolinensis. I. A description based on histology and autoradiography. J. Exp. Zool., 171:127-150.

Dennis, M.J., and J.W. Yip (1978) Formation and elimination of foreign synapses on adult salamander muscle. J. Physiol., 274:299-310.

Egar, M., and M. Singer (1972) The role of ependyma in spinal cord regeneration in the urodele Triturus. Exp. Neurol., $37: 422-430$.

Francis, E.T.B. (1934) The Anatomy of the Salamander. Oxford University Press, London.

Garcia, L., M. Verdiere-Sahugué, P.A. Drefus, M. Nicolet, and F. Rieger (1988) Association of tailed acetycholinesterase to lipidic membranes in mammalian skeletal muscle. Neurochem. Inst. 13, 402:231-236.

Géraudie, J., R. Nordlander and M. Singer (1989) The origins of spinal ganglia in the amphibian tail. In: Recent Trends in Regeneration Research, eds. V. Kiortsis, S. Koussoulakos and H. Wallace. Plenum Press, N.Y., pp. 237-249.

Goshgarian, H.G. (1977) A rapid silver impregnation for central and peripheral nerve fibers in paraffin and frozen sections. Exp. Neurol., 57:296-301.
Gulati, A.K., A.A. Zalewski, and A.M. Reddi (1983a) An immunofluorescent study of distribution of fibronectin and laminin during limb regeneration in the adult newt. Dev. Biol., 96:355-365.

Gulati, A.K., A.M. Reddi, and A.A. Zalewski (1983b) Changes in basement membrane zone components during skeletal muscle fiber degeneration and regeneration. J. Cell Biol., 97:957-963.

Iten, L.E., and S.V. Bryant (1976a) Stages of tail regeneration in the adult newt, Notophthalmus viridescens. J. Exp. Zool., 196:283-292.

Iten, L.E., and S.V. Bryant (1976b) Regeneration from different levels along the tail of the newt, Notophthalmus virides cens. J. Exp. Zool., 196:293-306.

Lappin, R.I., and L.L. Rubin (1985) Molecular forms of acetylcholinesterase in Xenopus muscle. Dev. Biol., 110:269-274.

Liu, H.C., and R.B. Maneely (1968) The development of the motor end-plates in embryonic and regenerative tail of Hemidactylus bowrengi. Acta Anat., 71:249-267.

Massoulié, J., and S. Bon (1982) The molecular forms of cholinesterase and acetylcholinesterase in vertebrates. Ann. Rev. Neurosci., 5:57-106.

Moody-Corbett, F., and M.W. Cohen (1982) Increased adhesiveness at sites of high acetylcholine receptor density in embryonic muscle cell cultures without nerve. J. Embryol. Exp. Morph., 72:53-69.

Mufti, S.A. (1973) Tail regeneration following amputation in adult Triturus viridescens. Pakistan J. Zool., 5:31-49.

Nicolet, M., L. Garcia, P.A. Dreyfus, M. Verdiere-Sahuqué, M. Pinçon-Raymond, and F. Riega (1987) Hydrophilic and hydrophobic attachment of both globular and asymetric acetylcholinesterase to froy muscle lamina sheaths. Neurochem. Inst., 11:189-198.

Nordlander, R.H., and M. Singer (1978) The role of ependyma in regeneration of the spinal cord of the urodele amphibian tail. J. Comp. Neurol., 180:349-374.

Nordlander, R.H., J.F. Singer, R. Beck, and M. Singer (1981) An ultrastructural examination of early ventral root formation in amphibia. J. Comp. Neur., 199:535-551.

Riou, J.F., T. Darribere, D.L. Shi, V. Richoux, and J.C. Boucaut (1987) Synthesis of laminin related polypeptides in oocytes, eggs and early embryos of the amphibian Pleurodeles waltl. Wilhelm Roux's Arch. Dev. Biol., 196:328-332.

Samuel, E.P. (1953) Towards controllable silver staining. Anat. Rec., 116:511-517.

Sanes, J.R. (1989) Extracellular matrix molecules that influence neural development. Ann. Rev. Neurosci., 12:491-516.

Singer, M. (1965) A theory of the trophic nervous control of amphibian limb regeneration, including a re-evaluation of quantitative nerve requirements. In Regeneration and Related Problems. V. Kiortsis and H.L.A. Trampusch, eds. North-Holland Publ. Co., Amsterdam, pp. 20-32.

Wallace, B.G., R.M. Nitkin, N.E. Reist, J.R. Fallon, N.N. Moayeri, and U.J. McMahan (1985) Aggregates of acetycholinesterase induced by acetylcholine receptor-aggregating factor. Nature, 315:574-577. 